\title{
Investigation of the Elastic Properties of Graphenylene Using Molecular Dynamics Simulations
}

\author{
Saeed Rouhi ${ }^{*}$, Ali Ghasemi \\ ${ }^{a}$ Young Researchers and Elite Club, Langarud Branch, Islamic Azad University, Langarud, Guilan, Iran \\ ${ }^{b}$ Department of Mechanical Engineering, Faculty of Engineering, Tehran North Branch, Islamic Azad \\ University, Tehran, Iran
}

Received: December 6, 2015; Revised: September 6, 2016; Accepted: October 20, 2016

\begin{abstract}
Molecular dynamics simulations are used here to study the mechanical behavior of graphenylene under uni-directional and bi-directional loadings. The effects of nanosheet chirality and size on Young's modulus of graphenylene are investigated. Compared to graphene, graphenylene possess a smaller elastic modulus. It is shown that for large armchair and zigzag graphenylenes, the effect of nanosheet size on the mechanical properties can be neglected. It is observed that increasing temperature results in decreasing Young's modulus of graphenylene. Besides, fracture of graphenylene occurs at large strains. Moreover, it is represented that for small graphenylenes, bulk modulus is significantly sensitive to the size variation. However, this sensitivity disappears for large nanosheets.
\end{abstract}

Keywords: Molecular dynamics simulations, Graphenylene, Young's modulus, Bulk modulus

\section{Introduction}

Since the discovery of graphene by Novoselov et al. in $2004^{1}$, this carbon allotrope has attracted the attention of many researchers. This can be attributed to its great physical, chemical, and mechanical properties ${ }^{1-3}$. Due to these great properties, graphene has found potential applications in several areas such as nanoelectronics, sensors, transistors, batteries, as well as polymer composites ${ }^{4-6}$.

Caused by these vast potential applications of graphene and carbon nanotubes (CNTs) in addition to various hybridized states of carbon ( $\mathrm{sp}, \mathrm{sp}^{2}$, and $\mathrm{sp}^{3}$ ), many researchers have devoted their investigations on finding other carbon allotropes which can possess extraordinary physical properties ${ }^{7-13}$. Graphenylene which was first described by Balaban et al. ${ }^{14}$ is one of these allotropes that some of the research works have investigated its properties ${ }^{15-23}$. The structure of graphenylene (so-called biphenylene carbon (BPC)) has been theoretically predicted $^{24-26}$ and synthesized ${ }^{27}$.

Recently, Song et al. ${ }^{22}$ studied the properties of graphenylene as the first example of a non-delocalized $\mathrm{sp}^{2}$-carbon structure. They showed that graphenylene has periodic pores with the diameters of $3.2 \AA$. Besides, it was claimed that it is a semiconductor with a narrow direct band gap which leads to its applicability in electronic devices and efficient hydrogen separation. Using ab initio density functional theory (DFT) calculations, $\mathrm{Yu}^{23}$ illustrated that graphenylene can store lithium with great density of energy. He showed that graphenylene can adsorb a lithium atom stronger than pristine graphene. Hankel and Searles ${ }^{28}$ showed that the lithium storage capacity of graphenylene is larger than graphite. Andrew T. Koch et al. ${ }^{29}$ used DFT and tight-binding methods to investigate the graphenylene based nanotubes.

*e-mail: s_rouhi@iaul.ac.ir
Mechanical properties of graphenylene are studied here by employing molecular dynamics (MD) simulations. To this end, the effect of some parameters such as chirality and size on Young's and bulk moduli of graphenylene are investigated. Besides, the fracture process of armchair and zigzag nanosheets under uni-directional and bi-directional tensions are explored.

\section{Models}

The structure of a sample graphenylene is shown in Figure 1. To obtain Young's modulus of graphenylene, the loads are applied in two directions, namely armchair and zigzag, which are shown schematically in Figure 1. As it is seen in Fig 1(b) and (c), for zigzag graphenylene, more $\mathrm{C}-\mathrm{C}$ bonds are directed along the loading direction (the ellipses have been used to show the directed bonds along the loading direction).

\section{Simulation method}

To obtain the mechanical properties of graphenylene, MD simulations are performed by LAMMPS MD code ${ }^{30,31}$. To define the interactions between the carbon atoms in the structure of graphenylene, Adaptive Intermolecular Reactive Empirical Bond Order (AIREBO) potential function ${ }^{32}$ is employed. This potential function is the improved version of Brenner's second-generation reactive empirical bond order potential $^{33}$ in which the Lennard-Jones term has been added to include van der Waals interactions. Moreover, torsional term is included to consider torsions of $\sigma$ bonds. So it is anticipated to give more accurate results than Brenner's 


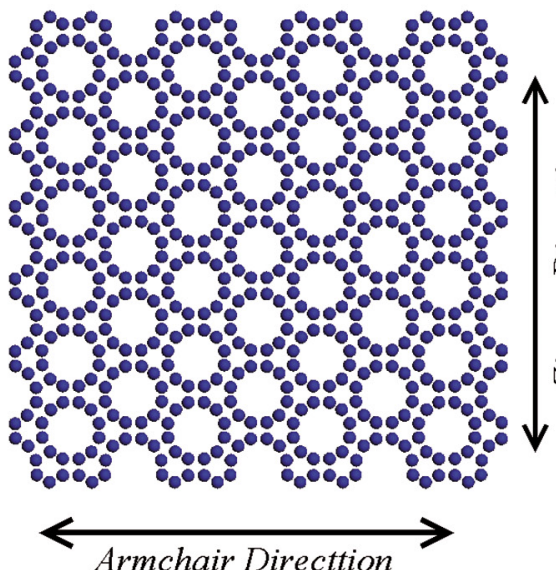

(a)

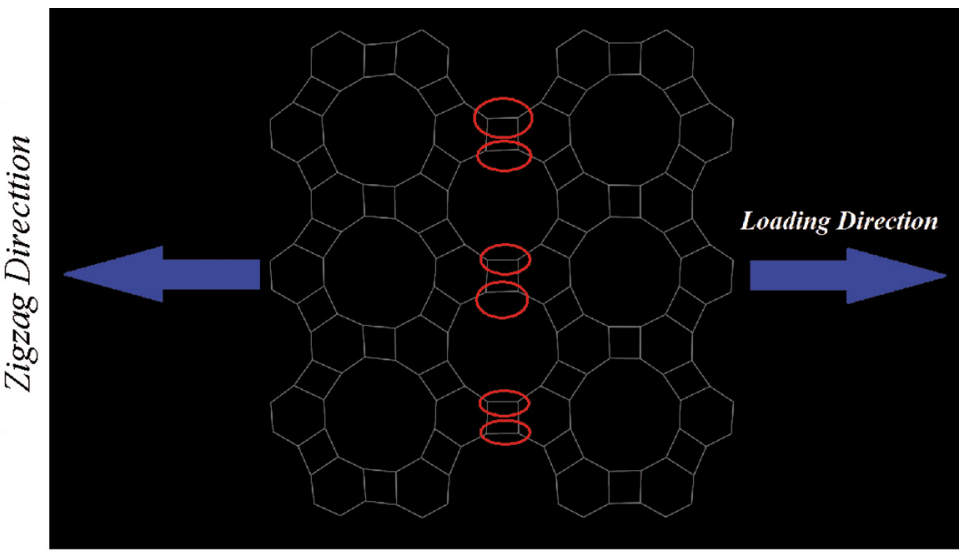

(b)

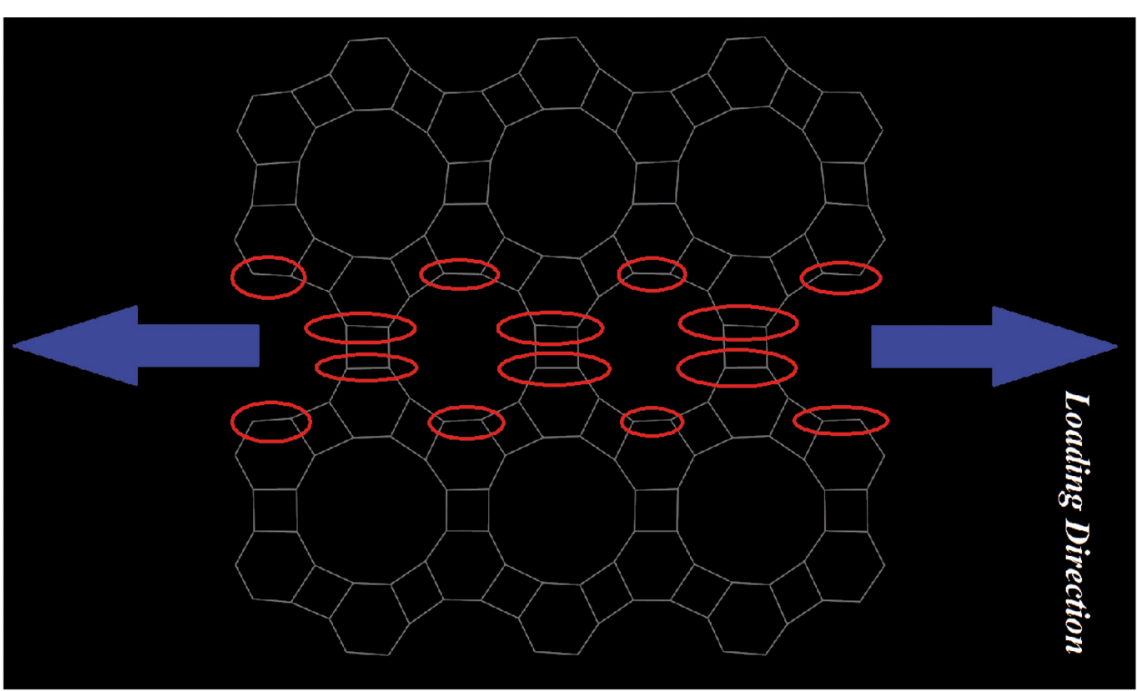

(c)

Figure 1: (a) Schematic of graphenylene with the armchair and zigzag direction, (b) Loading direction and C-C bonds detected along the loading direction for armchair and (c) zigzag nanosheets

potential function.After initial minimization of the system energy, the systems are relaxed for 20ps with the timestep of $1 f s$. For integration the equations of motion, velocity Verlet algorithm is utilized.

\section{Results and discussion}

To study the effect of atomic structure on the mechanical properties of graphenylene, armchair and zigzag nanosheets are modeled here. Moreover, considering different side lengths (a) and widths (b), the effect of graphenylene geometry on the mechanical properties are studied. Figure 2 shows armchair and zigzag nanosheets with the corresponding geometrical parameters. The loads are applied on the upper and lower edges of nanosheets. In each loading step, the boundary atoms are displaced and then the systems are let to relax for 2 ps ( 2000 steps with the time step of $1 \mathrm{fs})$. So, the stress would be distributed all over the nanosheets and the quasistatic condition of the tensile tests are simulated. All of the simulations are performed under NVT (constant number of molecules, constant volume, and constant temperature) ensemble. Besides, to ensure the stability of the simulations, Nose-Hoover thermostat is employed here.

\subsection{Young's modulus}

To obtain the mechanical properties of graphenylene, the lower sides of the nanosheets are fixed and upper sides are displaced incrementally. In each step, the strain and the system energy are recorded. As a unique value has not been reported for the thickness of nanostructures ${ }^{34-37}$, the in-plane Young's modulus is used here which is obtained as $^{38}$ :

$$
Y_{S}=\frac{1}{A_{0}}\left(\frac{\partial^{2} E_{S}}{\partial \varepsilon^{2}}\right)
$$




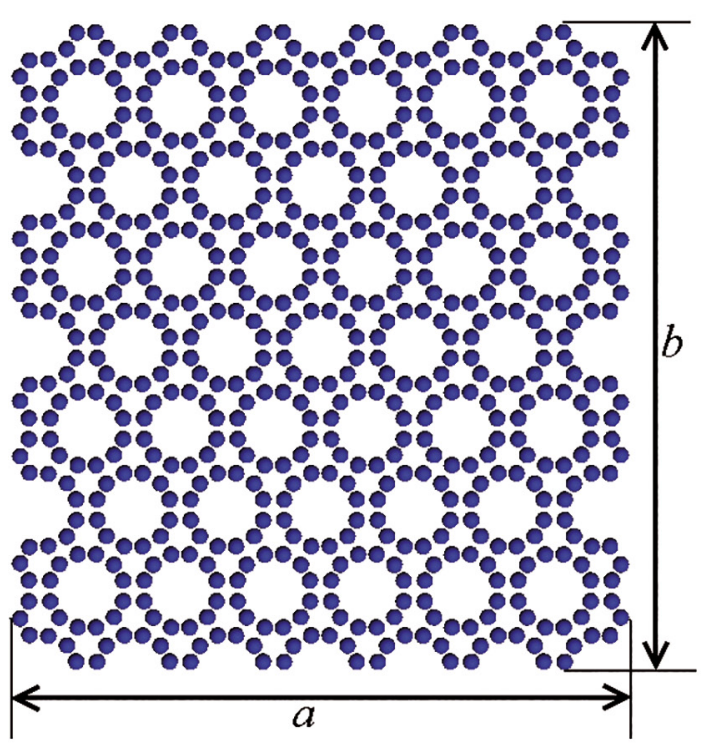

(a)

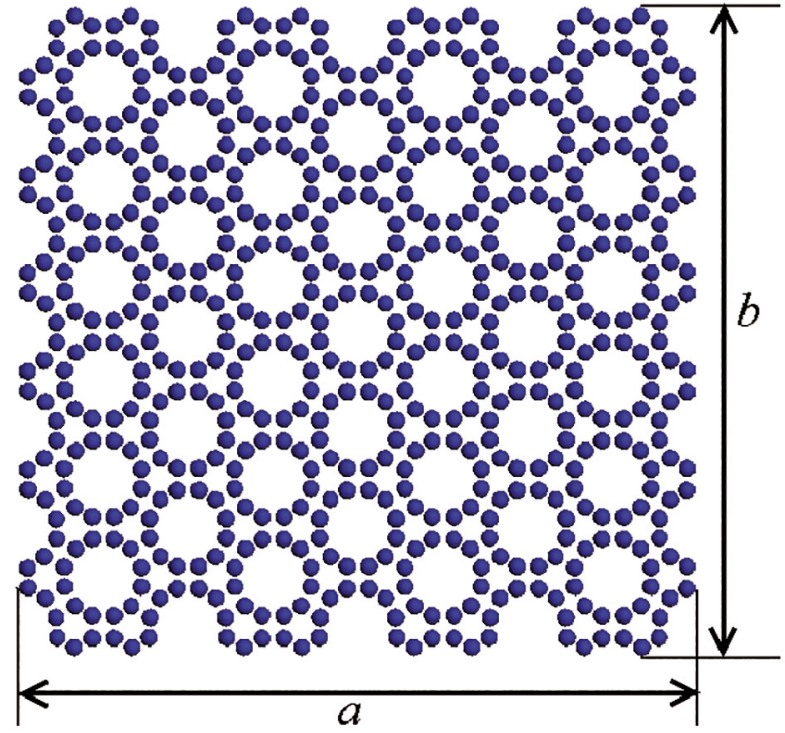

(b)

Figure 2: (a) Armchair and (b) zigzag graphenylene with the corresponding geometrical parameters

where $E_{S}, A_{0}$ and $\varepsilon$ are strain energy of system, initial unit cell area and strain, respectively. The recorded strain energies of armchair and zigzag graphenylenes with the dimensions of $30 \times 50 \AA^{2}$ are shown in Figs. 3 and 4 against strain. The drops of the curves show the bond breakages. As it is seen, the fracture occurs in different steps. In each step, some of the bonds break. This fact can be verified with the graphs of temperature versus strain which are shown in Figs. 5 and 6. As it is seen, each bond breakage results in a pick in the temperature of the systems.

The gradual breakage of the armchair and zigzag nanosheets are also represented in Figs. 7 and 8. It is seen that in each fracture step, some of the bonds are broken down. Finally, by connecting the cut bonds, a line of atoms is formed and stretched. For both of the nanosheets, the boundary effect is clearly seen. For armchair graphenylene, this effect results in breakage near the end of nanosheet. However, for zigzag graphenylene, although the some of the bonds near the left boundary are broken, the final fracture occurs at the middle of nanosheet. Moreover, comparing the figures at different steps, one can conclude that at each step, by breaking some bonds, some other new bonds are formed. The large fracture strain of graphenylene can be attributed to the formation of these new bonds.

Figure 9 depicts in-plane Young's moduli of armchair and zigzag graphenylenes with the aspect ratio of 1.5 versus nanosheet side length. The sizes of the considered nanosheets are given in Table 1. Since the dimensions can not be completely equal, the closest dimensions

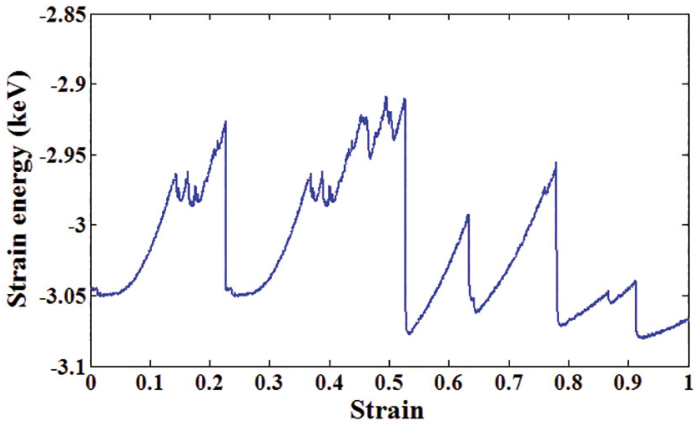

Figure 3: Strain energy of a $30 \times 50 \AA^{2}$ armchair graphenylene versus strain

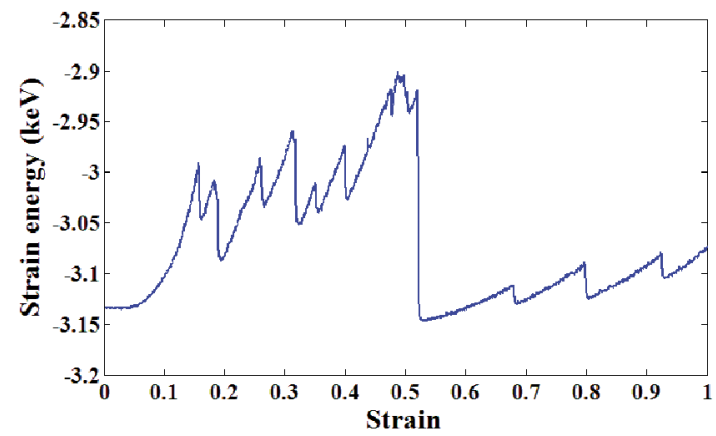

Figure 4: Strain energy of a $30 \times 50 \AA^{2}$ zigzag graphenylene versus strain.

are considered. As it is seen at a same size, zigzag graphenylenes have larger elastic modulus than armchair ones. However, the difference can be neglected for side lengths larger than $50 \AA$. Besides, increasing side length of graphenylene at constant aspect ratio result 


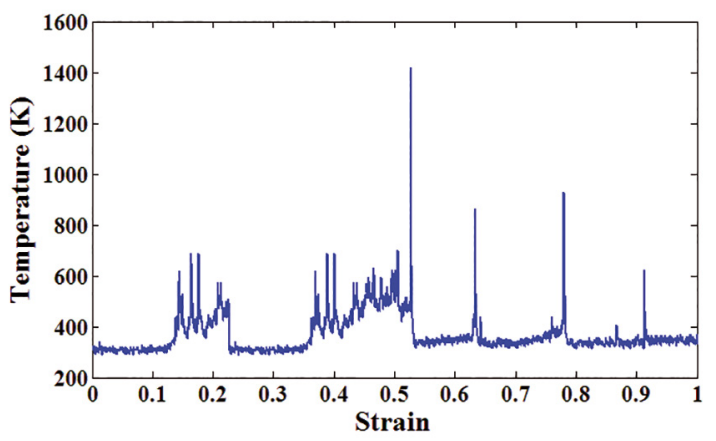

Figure 5: Temperature of a $30 \times 50 \AA^{2}$ armchair graphenylene versus strain.

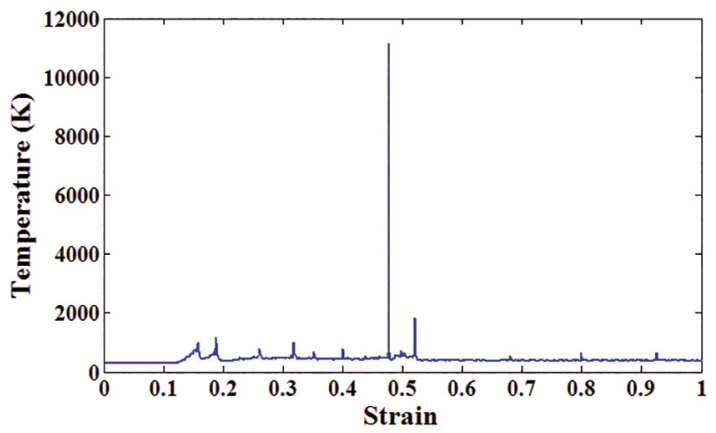

Figure 6: Temperature of a $30 \times 50 \AA^{2}$ zigzag graphenylene versus strain in decreasing the elastic modulus. The effect of side length on the elastic modulus also disappears for the side lengths larger than $60 \AA$.

Represented in Figure 10 are in-plane Young's moduli of armchair and zigzag graphenylene with the side length of $50 \AA$ versus graphenylene aspect ratio. The exact sizes of the considered nanosheets are given in Table 2. As the previous figure, it is seen that increasing aspect ratio of the nanosheets results in decreasing Young's modulus. Both of the effects of aspect ratio and chirality (being armchair and zigzag) diminishes for the aspect ratios larger than 2.

Young's moduli of $30 \times 50 \AA^{2}$ armchair and zigzag graphenylenes are obtained as $68.31 \mathrm{~N} / \mathrm{m}$ and $79.42 \mathrm{~N} / \mathrm{m}$, respectively, which are approximately $25 \%$ of Young's modulus of graphene $(272 \mathrm{~N} / \mathrm{m})$. The ultimate strength of armchair and zigzag $30 \times 50 \AA^{2}$ graphenylenes are $429.43 n N$ and $1140.59 n N$, respectively. Comparing these values with the ultimate strength of graphene $(1070 \mathrm{nN})$, it is seen that while the ultimate strength of armchair graphenylene is $40 \%$ of graphene, zigzag graphenyle has a larger ultimate strength than graphene. Besides, the large difference between the ultimate strength of armchair and zigzag nanosheet is interesting.
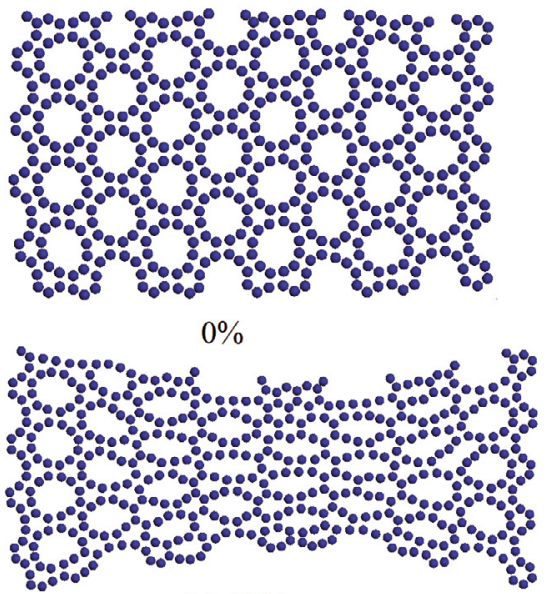

$27.53 \%$

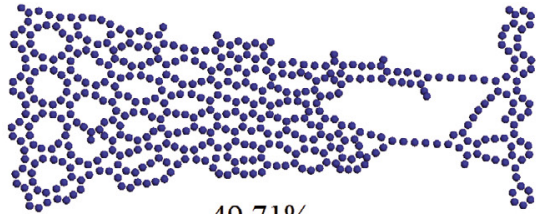

$49.71 \%$

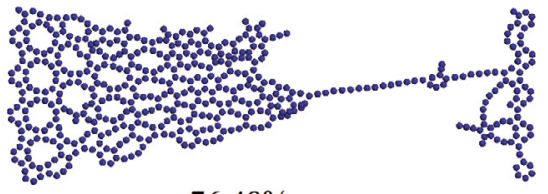

$76.48 \%$

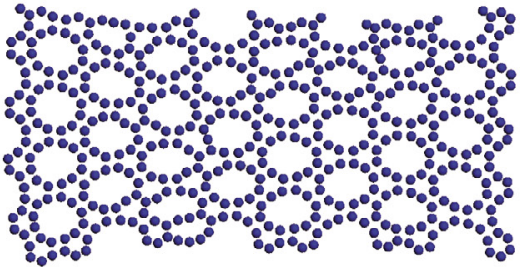

$15.30 \%$

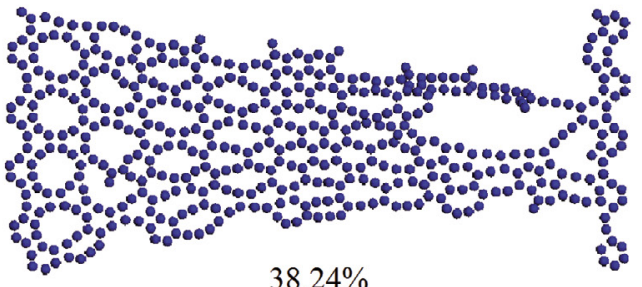

$38.24 \%$

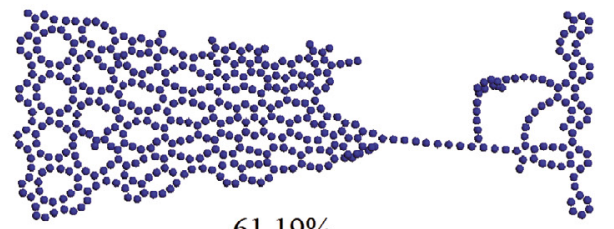

$61.19 \%$

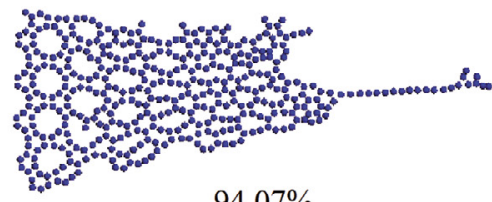

$94.07 \%$

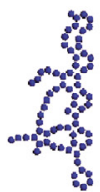

8

Figure 7: The shapes of a $30 \times 50 \AA^{2}$ armchair graphenylene at different strains 

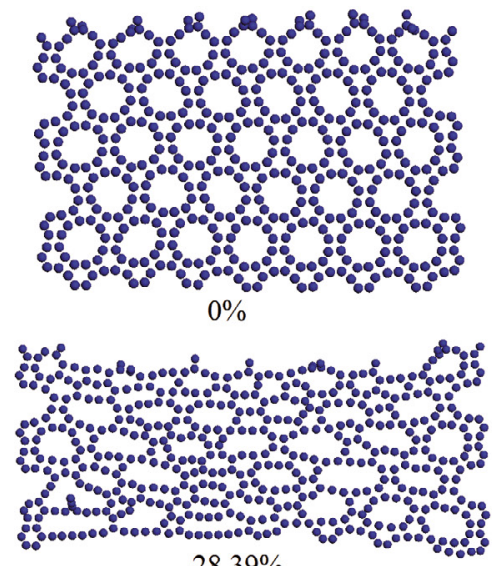

$28.39 \%$
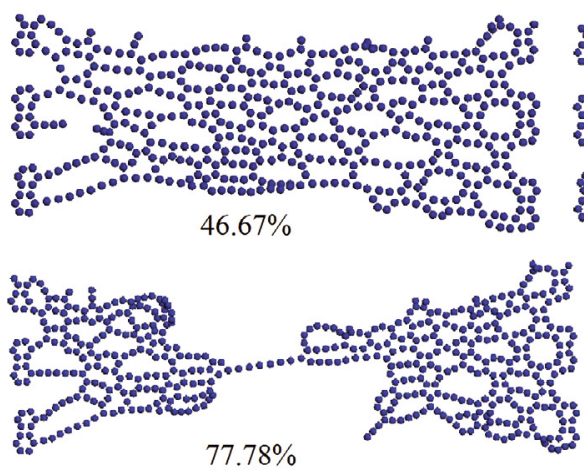

$77.78 \%$

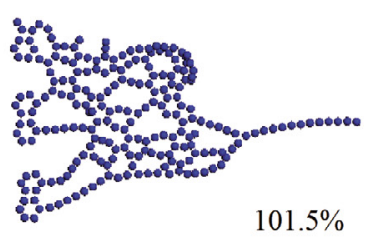

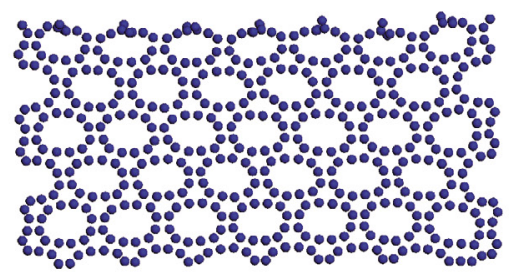

$11.67 \%$

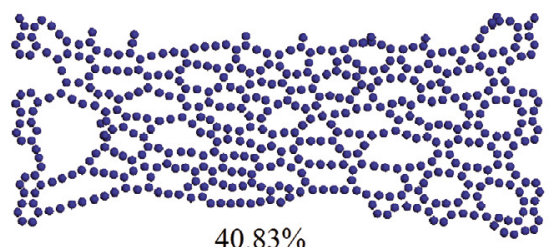

$40.83 \%$
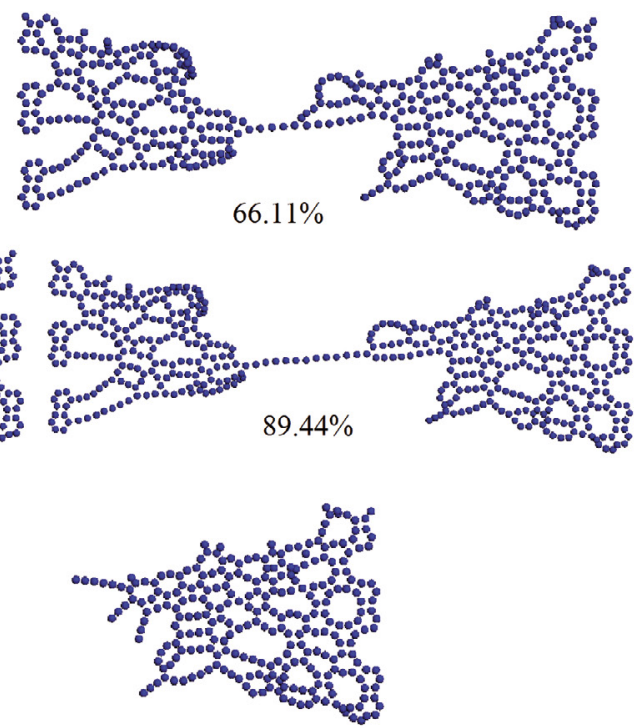

Figure 8: The shapes of a $30 \times 50 \AA^{2}$ zigzag graphenylene at different strains

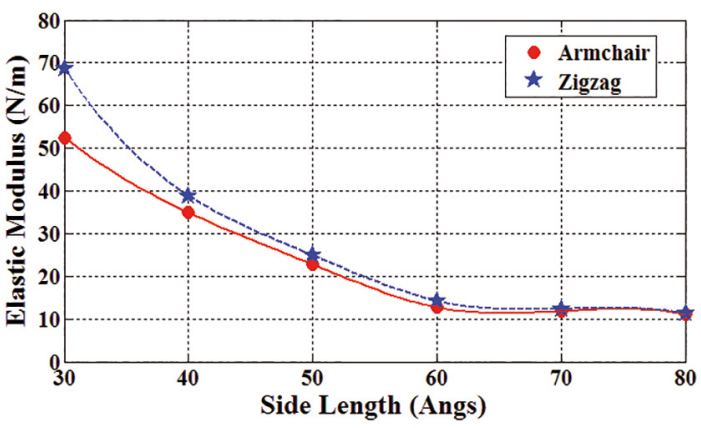

Figure 9: In-plane Young's modulus of armchair and zigzag graphenylenes with the aspect ratio of 1.5 versus graphenylene side length.

The yield and ultimate strains of $30 \times 50 \AA^{2}$ armchair graphenylene are obtained as $14.43 \%$ and $94.46 \%$, respectively. These values are $15.43 \%$ and $101.26 \%$ for zigzag graphenylene. Note to the large ultimate strains of armchair and zigzag graphenylene which were shown schematically in Figs. 7 and 8. Poisson's ratio of armchair and zigzag graphenylenes were obtained as $55 \%$ and $73 \%$, respectively which are smaller than Poisson's ratio of graphene (approximately $88 \%$ ).

The effect of temperature on the elastic modulus of the grapheneylene is given in Figure 11 for a $40 \times 60 \AA^{2}$ armchair nanosheet. Unlike Figs. 10 and 11 in which the dependence of the grapheneylene elastic modulus to the nanosheet side length and aspect ratio were investigated, no uniform behavior is observed by increasing the temperature. Therefore, a linear interpolation is used to view the general trace of the graphenylene elastic modulus by time variation. It is observed that nanosheets possess smaller Young's modulus at larger temperature. It can 
Table 1: Dimensions of armchair and zigzag nanosheets with the aspect ratio of 1.5

\begin{tabular}{lc}
\hline Zigzag & Armchair \\
\hline $32.4594 \times 46.0895 \AA^{2}$ & $30.4195 \times 45.3500 \AA^{2}$ \\
$39.4964 \times 60.1915 \AA^{2}$ & $40.5600 \times 61.7264 \AA^{2}$ \\
$51.2034 \times 75.0935 \AA^{2}$ & $50.1530 \times 74.6174 \AA^{2}$ \\
$61.7264 \times 90.0300 \AA^{2}$ & $60.1915 \times 90.9934 \AA^{2}$ \\
$73.4332 \times 105.5130 \AA^{2}$ & $70.4330 \times 103.8850 \AA^{2}$ \\
$80.4710 \times 120.4495 \AA^{2}$ & $80.4715 \times 120.2604 \AA^{2}$ \\
\hline
\end{tabular}

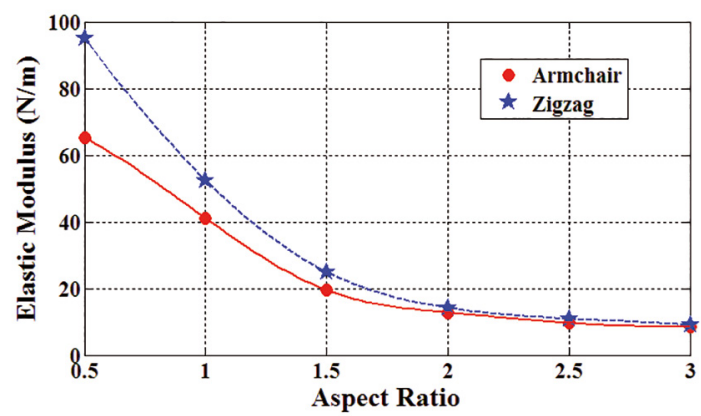

Figure 10: In-plane Young's modulus of armchair and zigzag graphenylenes with the side length of $50 \AA$ versus graphenylene aspect ratio.

Table 2: Dimensions of armchair and zigzag nanosheets with the aspect side length of $50 \AA$

\begin{tabular}{lc}
\hline Zigzag & Armchair \\
\hline $51.2034 \times 25.8095 \AA^{2}$ & $50.1530 \times 26.6060 \AA^{2}$ \\
$51.2034 \times 50.1530 \AA^{2}$ & $50.1530 \times 51.2034 \AA^{2}$ \\
$51.2034 \times 75.0935 \AA^{2}$ & $50.1530 \times 74.6174 \AA^{2}$ \\
$51.2034 \times 100.1695 \AA^{2}$ & $50.1530 \times 102.7002 \AA^{2}$ \\
$51.2034 \times 125.0595 \AA^{2}$ & $50.1530 \times 126.1138 \AA^{2}$ \\
$51.2034 \times 150.8700 \AA^{2}$ & $50.1530 \times 150.7130 \AA^{2}$ \\
\hline
\end{tabular}

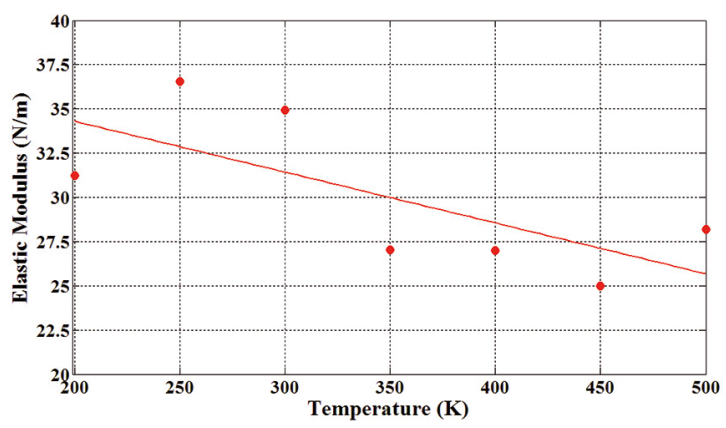

Figure 11: Effect of temperature on the elastic modulus of the grapheneylene for a $40 \times 60 \AA^{2}$ armchair nanosheet. be said that increasing the temperature results in higher kinetic energies of the atoms of nanosheets which results in smaller strength of nanosheets.

\subsection{Bulk modulus}

Bulk moduli of graphenylene are computed by bi-directional loading on nanosheets. Here, one horizontal edge and one vertical edge are constrained and the loads are applied in the form of displacement to the opposite edges. The following relation can be used to obtain the nanosheet bulk modulus:

$$
B=A_{0}\left(\frac{\partial^{2} E_{S}}{\partial A^{2}}\right)
$$

in which $A$ is the nanosheet area. The configurations of a $50 \times 50 \AA^{2}$ graphenylene under bi-directional loading at different timesteps are given in Figure 12. It is seen that the fracture initiates at the corners of the nanosheet and then propagates linearly parallel to the graphenylene boundaries. Linear propagation of fracture leads to breaking some bonds and formation some new bonds. Interestingly, bond breakage and formation of new bonds happen in the center of the nanosheet which is not in the fracture region. Besides, the fracture happens perpendicular to the zigzag direction and near the graphenylene boundary.

The computed bulk moduli of square graphenylenes with different side lengths are given in Figure 13. Table 3 represents the exact sizes of the selected nanosheets.It is seen that the effect of side length on the graphenylene bulk moduli is significant at the small side lengths. However, after $100 \AA$, the graph take a linear form and the effect of graphenylene size on the bulk modulus can be neglected.

\section{Conclusions}

Employing MD simulations, mechanical properties of graphenylene were computed. Applying unidirectional displacement on the armchair and zigzag graphenylene, Young's moduli of nanosheets with different sizes were obtained. It was shown that at a same size, zigzag graphenylenes possess larger Young's moduli than armchair. The effect of chirality was disappeared for large graphenylenes. Tracking the fracture process of armchair and zigzag graphenylenes, it was seen that the fracture happens at different steps in each of them some bonds are destroyed and some new ones are created. The boundary effect was observed for all unidirectional and bi-directional loading of graphenylenes. Finally, the bulk moduli of square graphenylenes were computed. 


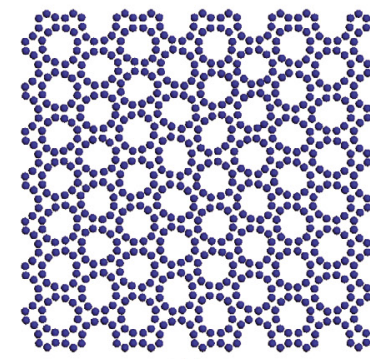

$0 \%$

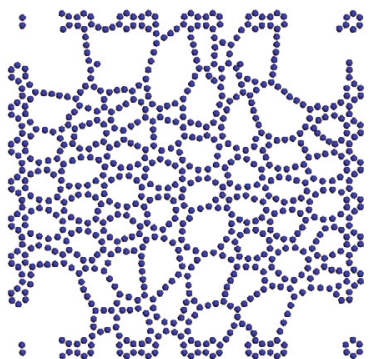

$19.41 \%$

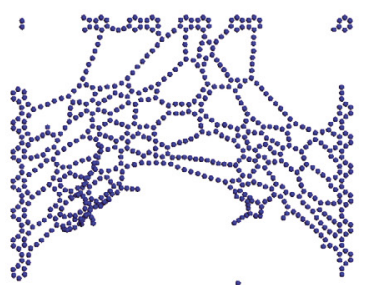

$=\quad \frac{1}{20}$ $42.67 \%$

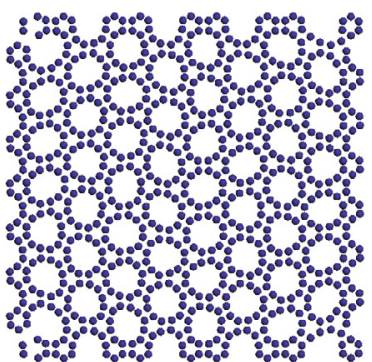

$3.91 \%$

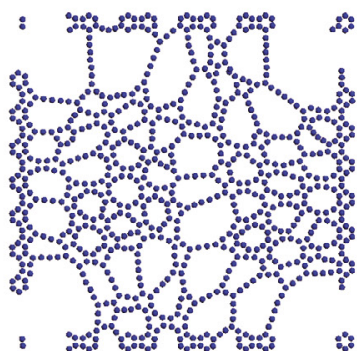
$27.16 \%$
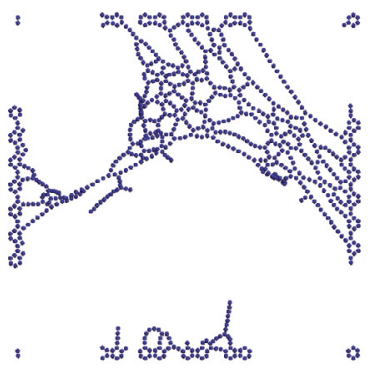

$58.17 \%$

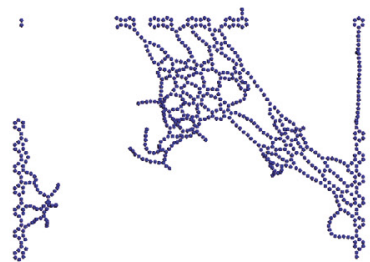

and

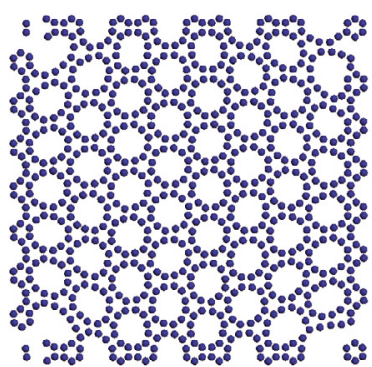

$11.66 \%$

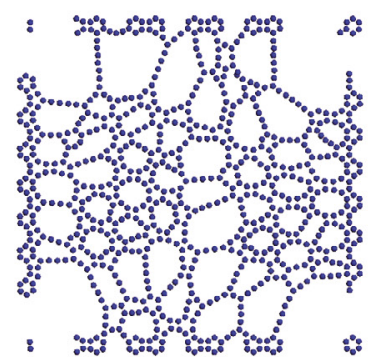

$34.91 \%$

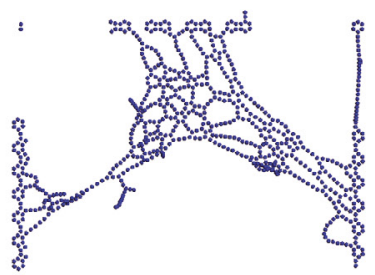

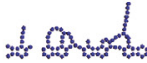
$77.54 \%$

Figure 12: The shapes of a $50 \times 50 \AA^{2}$ graphenylene under bi-directional loading at different strains

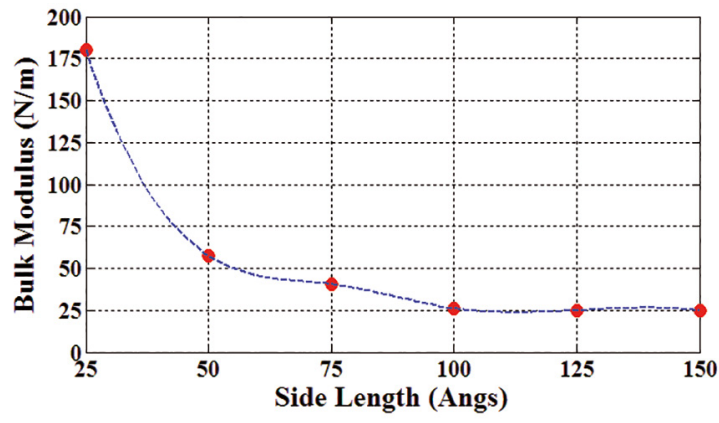

Figure 13: Computed bulk moduli of square graphenylenes with different side lengths
Table 3: Dimensions of selected nanosheets for investigation the bulk modulus

$26.6060 \times 25.8095 \AA^{2}$

$51.2034 \times 50.1530 \AA^{2}$

$74.6174 \times 75.0935 \AA^{2}$

$102.7002 \times 100.1695 \AA^{2}$

$126.1138 \times 125.0595 \AA^{2}$

$150.7130 \times 150.8700 \AA^{2}$ 


\section{References}

1. Novoselov KS, Geim AK, Morozov SV, Jiang D, Zhang Y, Dubonos SV, et al. Electric Field Effect in Atomically Thin Carbon Films. Science. 2004;306(5696):666-669.

2. Novoselov KS, Geim AK, Morozov SV, Jiang D, Katsnelson MI, Grigorieva IV, et al. Two-dimensional gas of massless Dirac fermions in graphene. Nature. 2005;438:197-200.

3. Ferrari AC, Meyer JC, Scardaci V, Casiraghi C, Lazzeri M, Mauri F, et al. Raman spectrum of graphene and graphene layers. Physics Review Letters. 2006;97(18):187401.

4. Novoselov KS, Jiang D, Schedin F, Booth TJ, Khotkevich VV, Morozov SV, et al. Two-dimensional atomic crystals. Proceedings of the National Academy of Sciences of the United States of America. 2005;102(30):10451-10453.

5. Geim AK, Novoselov KS. The rise of graphene. Nature Materials. 2007;6:183-191.

6. Mohanty N, Berry V. Graphene-based single-bacterium resolution biodevice and DNA transistor: interfacing graphene derivatives with nanoscale and microscale biocomponents. Nano Letters. 2008;8(12):4469-4476.

7. Kroto HW, Heath JR, O'Brien SC, Curl RF, Smalley RE. $\mathrm{C}_{60}$ : Buckminsterfullerene. Nature. 1985;318:162-163.

8. Kong XY, Ding Y, Yang R, Wang ZL. Single-Crystal Nanorings Formed by Epitaxial Self-Coiling of Polar Nanobelts. Science. 2004;303(5662):1348-1351.

9. Narita N, Nagai S, Suzuki S, Nakao K. Optimized geometries and electronic structures of graphyne and its family. Physical Review B. 1998;58(16):11009.

10. Narita N, Nagai S, Suzuki S, Nakao K. Electronic structure of three-dimensional graphyne. Physical Review $B$. 2000;62(16):11146.

11. Narita N, Nagai S, Suzuki S. Potassium intercalated graphyne. Physical Review B. 2001;64(24):245408.

12. Sheng XL, Yan QB, Ye F, Zheng QR, Su G. T-carbon: a novel carbon allotrope. Physical Review Letters. 2011;106(15):155703.

13. Minyaev RM, Avakyan VE. Supertetrahedrane-A new possible carbon allotrope. Doklady Chemistry. 2010;434(2):253-256.

14. Balaban AT, Rentea CC, Ciupitu E. Chemical graphs. VI. Estimation of the relative stability of several planar and tridimensional lattices for elementary carbon. Revue Roumaine de Chimie. 1968;13:231-247.

15. Balaban AT, Vollhardt KPC. Heliphenes and Related Structures. The Open Organic Chemistry Journal. 2011;5:117-126.

16. Balaban AT. Carbon and its nets. Computers \& Mathematics with Applications. 1989;17(1-3):397-416.

17. Balaban AT, Klein DJ, Folden CA. Diamond-graphite hybrids. Chemical Physics Letters. 1994;217(3):266-270.

18. Zhu H, Balaban AT, Klein DJ, Živković TP. Conjugatedcircuit computations on two-dimensional carbon networks. The Journal of Chemical Physics. 1994;101(6):5281-5292.
19. Klein DJ, Balaban AT. Clarology for conjugated carbon nanostructures: Molecules, polymers, graphene, defected graphene, fractal benzenoids, fullerenes, nano-tubes, nano-cones, nano-tori, etc. The Open Organic Chemistry Journal. 2011;5(Suppl 1-M3):27-61.

20. Merz KM Jr., Hoffmann R, Balaban AT. 3,4-Connected carbon nets: through-space and through-bond interactions in the solid state. Journal of the American Chemical Society. 1987;109(22):6742-6751.

21. Diercks R, Vollhardt KPC. Tris(benzocyclobutadieno)benzene, the triangular [4]phenylene with a completely bond-fixed cyclohexatriene ring: cobalt-catalyzed synthesis from hexaethynylbenzene and thermal ring opening to $1,2: 5,6: 9,10$-tribenzo-3,4,7,8,11,12hexadehydro[12]annulene. Journal of the American Chemical Society. 1986;108(11):3150-3152.

22. Song Q, Wang B, Deng K, Feng X, Wagner M, Gale JD, et al. Graphenylene, a unique two-dimensional carbon network with nondelocalized cyclohexatriene units. Journal of Materials Chemistry C. 2013;1(1):38-41.

23. Yu YX. Graphenylene: a promising anode material for lithium-ion batteries with high mobility and storage Journal of Materials Chemistry A. 2013;1(43):13559-13566.

24. Baughman RH, Eckhardt H, Kertesz M. Structure-property predictions for new planar forms of carbon: Layered phases containing $s p^{2}$ and $s p$ atoms. The Journal of Chemical Physics. 1987;87(11):6687-6699.

25. Enyashin AN, Ivanovskii AL. Graphene allotropes. Physica Status Solidi (b). 2011;248(8):1879-1883.

26. Brunetto G, Autreto PAS, Machado LD, Santos BI, Santos RPB, Galvão DS. Nonzero Gap Two-Dimensional Carbon Allotrope from Porous Graphene. The Journal of Physical Chemistry $C$. 2012;116(23):12810-12813.

27. Schulman JM, Disch RL. A Theoretical Study of Large Planar $[N]$ Phenylenes. The Journal of Physical Chemistry A. 2007;111(39):10010-10014.

28. Hankel M, Searles DJ. Lithium storage on carbon nitride, graphenylene and inorganic graphenylene. Physical Chemistry Chemical Physics. 2016;18(21):14205-14215.

29. Koch AT, Khoshaman AH, Fan HD, Sawatzky GA, Nojeh A. Graphenylene Nanotubes. The Journal of Physical Chemistry Letters. 2015;6(19):3982-3987.

30. LAMMPS Molecular Dynamics Simulator. Available from: $<$ http://lammps.sandia.gov $>$. Access in: 1/3/2010.

31. Plimpton S. Fast Parallel Algorithms for Short-Range Molecular Dynamics. Journal of Computational Physics. 1995;117(1):1-19.

32. Stuart SJ, Tutein AB, Harrison JA. A reactive potential for hydrocarbons with intermolecular interactions. The Journal of Chemical Physics. 2000;112(14):6472-6486.

33. Brenner DW, Shenderova OA, Harrison JA, Stuart SJ, Ni B, Sinnott SB. A second-generation reactive empirical bond order (REBO) potential energy expression for hydrocarbons. JournaL of Physics: Condensed Matter. 2002;14(4):783-802. 
34. Xin Z, Jianjun Z, Zhong-can OY. Strain energy and Young's modulus of single-wall carbon nanotubes calculated from electronic energy-band theory. Physical Review $B$. 2000;62(20):13692-13696.

35. Tu ZC, Ou-Yang ZC. Single-walled and multiwalled carbon nanotubes viewed as elastic tubes with the effective Young's moduli dependent on layer number. Physical Review $B$. 2002;65(23):233407.
36. Pantano A, Parks DM, Boyce MC. Mechanics of deformation of single- and multi-wall carbon nanotubes. Journal of the Mechanics and Physics of Solids. 2004;52(4):789-821.

37. Goupalov SV. Continuum model for long-wavelength phonons in two-dimensional graphite and carbon nanotubes. Physical Review B. 2005;71(8):085420.

38. Topsakal M, Cahangirov S, Ciraci S. The response of mechanical and electronic properties of graphane to the elastic strain. Applied Physics Letters. 2010;96(9):091912. 